\title{
Gross 曲线的一些算术性质
}

\section{康云凌}

南京审计学院理学院, 南京 211815

E-mail: kangyl@nau.edu.cn

收稿日期: 2014-09-22；接受日期: 2014-12-15

国家自然科学基金 (批准号: 11401312)、江苏省高校自然科学研究面上项目 (批准号: 14KJB110012) 和江苏高校优势学科建设工 程资助项目

摘要 本文研究一类特殊 Gross 曲线的算术性质, 建立 Selmer 群以及增大的 Selmer 群和域扩张的 Galois 群之间的联系, 并刻画了曲线在局部域上有理点的部分结构信息.

关键词 椭圆曲线 复乘 Iwasawa 理论

MSC (2010) 主题分类 $11 \mathrm{R} 23,11 \mathrm{G} 40$

\section{1 前言}

设 $E$ 是定义在数域 $F$ 上的椭圆曲线, 以虚二次域 $K$ 的整数环 $\mathcal{O}$ 为复乘环, $F$ 包含 $K$ 且 $F\left(E_{\mathrm{torsion}}\right) / K$ 是交换域扩张. 满足这样条件的椭圆曲线称之为 Gross 曲线, 这类曲线具有丰富的算 术性质. 我们将在本文中总结一些和 Iwasawa 理论有关的定理, 并进一步研究 Gross 曲线的 Selmer 群 与增大的 Selmer 群, 刻画曲线有理点的部分结构.

我们使用 $p$-adic 方法, 结合以下正合列, 可以看出 Selmer 群的阶蕴含了沙群的阶的信息,

$$
0 \longrightarrow E(F) \otimes_{\mathcal{O}} K_{\mathfrak{p}} / \mathcal{O}_{\mathfrak{p}} \longrightarrow \operatorname{Sel}_{\mathfrak{p} \infty}(E / F) \longrightarrow \amalg_{\mathfrak{p} \infty} \longrightarrow 0 .
$$

同时椭圆曲线的 $\mathrm{CM}$ 理论给出了以下刻画:

(1) Selmer 群 $S=\operatorname{Sel}_{\mathfrak{p} \infty}(E / F)$ 和 Galois 群 $X_{\infty}$ 的 twist 对偶.

(2) $L(E / F, 1)=L\left(\psi_{E / F}, 1\right) L\left(\bar{\psi}_{E / F}, 1\right)$.

而 Iwasawa 主猜想又联系了 Selmer 群 $S$ 和 $L$ 函数特殊值 $L(E / F, 1)$, 因此在复乘情形, Iwasawa 主猜想会联系 Galois 群 $X_{\infty}$ 和 $L(\bar{\psi}, 1)$, 这将在下一节介绍.

\section{Iwasawa 主猜想}

首先我们介绍一些记号:

$K$ 是一个虚二次域, $\mathcal{O}$ 是 $K$ 的整数环, $H$ 是 $K$ 的 Hilbert 类域;

$p$ 是一个有理素数, 并且不整除 $H$ 的整数环中单位根的个数;

$\mathfrak{p}$ 是 $K$ 的整除 $p$ 的素元, $\mathfrak{p}^{*}$ 是 $\mathfrak{p}$ 的共轭素元; 
$K_{0}$ 是 $K$ 的有限交换扩张, $K_{0}$ 包含 $H$, 并且 $p \nmid\left[K_{0}: K\right]$;

$K_{\infty}$ 是 $K$ 的交换扩张, 包含 $K_{0}$, 并且 $\operatorname{Gal}\left(K_{\infty} / K_{0}\right) \cong \mathbb{Z}_{p}$ 或者 $\mathbb{Z}_{p}^{2}$;

令 $\Delta=\operatorname{Gal}\left(K_{0} / K\right), \Gamma=\operatorname{Gal}\left(K_{\infty} / K_{0}\right) \cong \mathbb{Z}_{p}$ 或 $\mathbb{Z}_{p}^{2}, \mathcal{G}=\operatorname{Gal}\left(K_{\infty} / K\right) \cong \Delta \times \Gamma$;

$F$ 为 $K_{\infty} / K$ 的一个子扩张;

$X(F)=\operatorname{Gal}(M(F) / F)$, 这里 $M(F)$ 是 $F$ 的在 $\mathfrak{p}$ 之外不分歧的极大交换 $p$ 扩张.

若 $[F: K]$ 是有限扩张, 则以下记号有意义:

$A(F)$ 表示 $F$ 的理想类群的 $p$ 部分;

$U(F)$ 表示 $F \otimes_{K} K_{\mathfrak{p}}$ 中, 模 $\mathfrak{p}$ 上的素元同余于 1 的局部单位构成的群;

$\overline{\mathcal{E}}(F)$ 表示 $\mathcal{E}(F) \cap U(F)$ 在 $U(F)$ 中的闭包, 其中 $\mathcal{E}(F)$ 是 $F$ 的整体单位群;

$\overline{\mathcal{C}}(F)$ 表示 $\mathcal{C}(F) \cap U(F)$ 在 $U(F)$ 中的闭包, 其中 $\mathcal{C}(F)$ 是 $F$ 的椭圆单位群.

当 $[F: K]$ 是无限扩张时, 我们将使用如下记号 $A(F), U(F), \overline{\mathcal{E}}(F)$ 和 $\overline{\mathcal{C}}(F)$, 分别表示对于 $K$ 的包 含在 $F$ 中的有限扩张关于范映射取对应的反向极限. 特别地, 当 $F=K_{\infty}$ 时我们使用 $A_{\infty}, \overline{\mathcal{E}}_{\infty}, \overline{\mathcal{C}}_{\infty}$, $U_{\infty}$ 等记号.

注 1 存在无穷多 $K_{0}$ 的 $\mathbb{Z}_{p^{-}}$扩张且在 $K$ 上是交换的, 但是根据 Leopoldt 猜想, 所有这些扩张 都包含在唯一的在 $K$ 上交换的 $K_{0}$ 的 $\mathbb{Z}_{p^{-}}^{2}$ 扩张当中.

整体类域论给出以下正合列:

$$
0 \longrightarrow \overline{\mathcal{E}}_{\infty} / \overline{\mathcal{C}}_{\infty} \longrightarrow U_{\infty} / \overline{\mathcal{C}}_{\infty} \longrightarrow X_{\infty} \longrightarrow A_{\infty} \longrightarrow 0 .
$$

我们定义 Iwasawa 代数 $\Lambda=\mathbb{Z}_{p}[[\mathcal{G}]]$, 则上述正合列同时也是一个 $\Lambda$ - 模的正合列.

设 $Y$ 是一个 $\mathbb{Z}_{p}[\Delta]$ - 模, $\chi$ 是 $\Delta$ 的一个不可约 $\mathbb{Z}_{p^{-}}$表示, 我们定义 $Y \chi$ 为 $Y$ 的 $\chi$ - 分支. 所以有 $\Lambda^{\chi} \cong R_{\chi}[[\Gamma]]$, 这里 $R_{\chi}$ 是 $\mathbb{Q}_{p}$ 的次数为 $\operatorname{dim}(\chi)$ 的不分歧扩张的整数环.

定理 2 上述正合列 $(2.1)$ 中的 $\Lambda$ - 模都是有限生成的, 其中 $\overline{\mathcal{E}}_{\infty} / \overline{\mathcal{C}}_{\infty}$ 和 $A_{\infty}$ 是 torsion 模, $X_{\infty}$ 没有非零的 pseudo-null 子模. 并且我们有如下结论:

(1) 设 $\mathfrak{p} \neq \mathfrak{p}^{*}$. 则 $U_{\infty} / \overline{\mathcal{C}}_{\infty}$ 和 $X_{\infty}$ 是 torsion 的, 并且

$$
\operatorname{Char}\left(A_{\infty}\right)=\operatorname{Char}\left(\overline{\mathcal{E}}_{\infty} / \overline{\mathcal{C}}_{\infty}\right), \quad \operatorname{Char}\left(X_{\infty}\right)=\operatorname{Char}\left(U_{\infty} / \overline{\mathcal{C}}_{\infty}\right)
$$

(2) 设 $\mathfrak{p}=\mathfrak{p}^{*}$. 若 $\chi$ 是 $\Delta$ 的一个不可约 $\mathbb{Z}_{p^{-}}$表示, 并且对于 $\mathfrak{p}$ 在 $\Delta$ 内的分解群, $\chi$ 在其上非平凡, 则我们有

$$
\operatorname{Char}\left(A_{\infty}\right)^{\chi}=\operatorname{Char}\left(\overline{\mathcal{E}}_{\infty} / \overline{\mathcal{C}}_{\infty}\right)^{\chi}
$$

证明 参见文献 [1].

为了完整表达主猜想, 我们需要联系本节定义的 Iwasawa 模和解析的对象 $p$-adic $L$ - 函数. 在下 一节我们回顾一下关于 $p$-adic $L$ - 函数的构造.

\section{$3 p$-adic $L$ - 函数}

本节中 $K, \mathcal{O}$ 和 $p \mathcal{O}=\mathfrak{p p}^{*}$ 等记号同上一节一样, 设 $\mathfrak{p} \neq \mathfrak{p}^{*}$. 固定 $K$ 的一个与 $\mathfrak{p}$ 互素的整理想 $\mathfrak{f}$ 使得 $w_{\mathfrak{f}}=1$, 这里 $w_{\mathfrak{f}}$ 表示模 $\mathfrak{f}$ 同余 1 的 $K$ 中的单位根的个数. 令 $F=K(\mathfrak{f}), F_{n}=K\left(\mathfrak{f} \mathfrak{p}^{n}\right)$. 固定一个 $(1,0)$ 型, 且导子整除 $\mathfrak{f}$ 的 Grössencharak $\varphi$, 选取定义在 $F$ 上的椭圆曲线 $E$ 满足:

(1) $E$ 以 $\mathcal{O}$ 为复乘环; 
(2) $F\left(E_{\text {torsion }}\right)$ 是 $K$ 的交换扩张;

(3) $\psi_{E / F}=\varphi \circ \mathrm{N}_{F / K}$, 这里 $\psi_{E / F}$ 是 $E / F$ 的 Grössencharak.

根据文献 [2, 第 41 页的引理], 满足上述条件的 $\varphi$ 和 $E$ 是存在的. 对于任意被 $\mathfrak{f}$ 整除的 $\mathfrak{g}$, $K(\mathfrak{g})=F(E(\mathfrak{g}))$, 特别地, 我们可以取 $\mathfrak{g}=\mathfrak{f p}^{n}$. 而且 $\operatorname{Gal}\left(F_{n} / F\right)$ 通过其在 $E_{\mathfrak{p}^{n}}$ 上的作用与 $\left(\mathcal{O} / \mathfrak{p}^{n}\right)^{\times}$同 构 (参见文献 $[2$, 第 44 页的推论 1.7$]$ ).

令

$$
\Phi=F \otimes_{K} K_{\mathfrak{p}}, \quad R=\mathcal{O}_{F} \otimes_{\mathcal{O}} \mathcal{O}_{\mathfrak{p}},
$$

并用 $F_{n}$ 替换 $F$ 即可类似定义 $\Phi_{n}$ 和 $R_{n}$. 令

$$
\mathcal{G}=\operatorname{Gal}\left(F_{\infty} / K\right), \quad G=\operatorname{Gal}\left(F_{\infty} / F\right)=\Gamma \times \Delta,
$$

这里 $\Gamma \cong 1+p \mathbb{Z}_{p}, \Delta \cong \mathbb{F}_{p}^{\times}$. 我们定义 $\mathcal{U}=\lim U_{n}$, 其中 $U_{n}$ 表示主单位.

现在我们可以定义 periods. 如果有必要可以将 $E$ 替换成其共轭, 所以不妨假设 $L=\Omega \mathfrak{f}, \Omega \in \mathbb{C}^{\times}$, 这里 $L$ 是选定的 Weierstrass 模型的周期格. 我们同样可以定义 $p$-adic period $\Omega_{\mathfrak{p}}$. 令

$$
F^{\prime}=F\left(E_{\mathfrak{p}^{* \infty}}\right), \quad R^{\prime}=\mathcal{O}_{F^{\prime}} \otimes_{\mathcal{O}} \mathcal{O}_{\mathfrak{p}}
$$

令 $\hat{R}$ 表示 $R^{\prime}$ 的完备化, 则 $\Omega_{\mathfrak{p}}$ 是 $\hat{R}^{\times}$中的一个元素. 而且我们可以典范的选取 $\Omega_{\mathfrak{p}}$ 使得 $\left\langle\Omega, \Omega_{\mathfrak{p}}\right\rangle \in$ $\mathbb{C}^{\times} \times \mathbb{C}_{p}^{\times}$在模掉 $F^{\times}$中在 $\mathfrak{p}$ 处为单位的那些元素构成的群的意义下定义良好. 具体细节可以参见文 献 $[2$, 第 67,68 和 75 页].

对任意导子整除 $\mathfrak{f p}^{\infty}$ 的 $(1,0)$ 型的 Grössencharak $\varepsilon$, 我们可以定义一个 “类 Gauss 和” $G(\varepsilon)$ 使其 在 $\mathfrak{p}$ 处为单位. 参见文献 [2, 第 75 页]. 最后我们引入如下两个定理, 完成 $p$-adic $L$ - 函数的构造.

定理 3 令 $\mathfrak{f}$ 为 $K$ 的一个非平凡的整理想, $\mathfrak{p}$ 是一个分裂的素除子且满足 $(\mathfrak{p}, \mathfrak{f})=1$. 则存在周期 $\Omega \in \mathbb{C}^{\times}$和 $\Omega_{\mathfrak{p}} \in \mathbb{C}_{p}^{\times}$, 以及唯一的定义在

$$
\mathcal{G}(\mathfrak{f})=\operatorname{Gal}\left(K\left(\mathfrak{f p}^{\infty}\right) / K\right)
$$

上 $p$-adic 整测度 $\mu(\mathfrak{f})$, 使得对于任意导子整除 $\mathfrak{f p}^{\infty}$ 的 $(1,0)$ 型的 Grössencharak $\varepsilon$,

$$
\Omega_{\mathfrak{p}}^{-1} \int_{\mathcal{G}(\mathfrak{f})} \varepsilon(\sigma) d \mu(\mathfrak{f})(\sigma)=\Omega^{-1} G(\varepsilon)\left(1-\frac{\varepsilon(\mathfrak{p})}{p}\right) L_{\mathfrak{f}}(\bar{\varepsilon}, 1) .
$$

若 $\mathfrak{f} \mid \mathfrak{g}, \bar{\mu}(\mathfrak{g})$ 是通过 $\mathcal{G}(\mathfrak{f})$ 上的测度 $\mu(\mathfrak{f})$ 诱导出的测度, 则

$$
\bar{\mu}(\mathfrak{g})=\mu(\mathfrak{f}) \prod\left(1-\sigma_{\mathfrak{l}}^{-1}\right),
$$

这里的乘积过所有整除 $\mathfrak{g}$ 但不整除 $\mathfrak{f}$ 的素除子 (计算重数).

若 $\mathfrak{f}=(1)$ 类似的结论也成立, 只是这种情况下 $\mu(1)$ 是一个伪测度, 对于任意 $\sigma \in \mathcal{G}(1),(1-\sigma) \mu(1)$ 是一个 $p$-adic 测度.

定理 4 (Yager) 设 $\mathfrak{f}$ 是 $K$ 的和 $p$ 互素的整理想. 对于 $D=\widehat{\mathbb{Z}_{p}^{n r}}$ 存在唯一的测度, 记作 $\mu\left(\mathfrak{f}^{\infty}\right)$, 属于 $\Lambda_{D}\left(G\left(K\left(\mathfrak{f} p^{\infty}\right) / K\right)\right)$, 使得对于任意导子整除 $\mathfrak{f} p^{\infty}$ 的 $(k, j)$ 型的 Grössencharak $\epsilon$, 这里 $0 \leqslant-j$ 并 且 $k>0$, 我们有

$$
\Omega_{p}^{j-k} \int_{G\left(K\left(\mathfrak{f p} p^{\infty}\right) / K\right)} \epsilon(\sigma) \mu\left(\mathfrak{f}^{\infty}\right)=\Omega_{\infty}^{j-k}(k-1) !\left(\frac{\sqrt{d_{K}}}{2 \pi}\right)^{j} G(\epsilon)\left(1-\frac{\epsilon(\mathfrak{p})}{p}\right) L_{\overline{\mathfrak{p}} \mathfrak{f}}\left(\epsilon^{-1}, 0\right),
$$


这里

$$
G(\epsilon):=\frac{\phi^{k} \bar{\phi}^{j}\left(\mathfrak{p}^{n}\right)}{p^{n}} G(\chi):=\frac{\phi^{k} \bar{\phi}^{j}\left(\mathfrak{p}^{n}\right)}{p^{n}} \sum_{\gamma \in M} \chi(\gamma)\left(\zeta_{n}^{\gamma}\right)^{-1},
$$

这里我们记 $\epsilon=\phi^{k} \bar{\phi}^{j} \chi, \phi$ 是某一个导子与 $\mathfrak{p}$ 互素的 $(1,0)$ 型的 Grössencharak, 而 $\chi$ 是一个有限阶的 特征, 在 $\mathfrak{p}$ 处的导子等于 $\mathfrak{p}^{n}$,

$$
M:=\left\{\gamma \in \operatorname{Gal}\left(K\left(\mathfrak{f} \overline{\mathfrak{p}}^{\infty} \mathfrak{p}^{n}\right) / K\right) \mid \gamma_{\mid F^{\prime}}=\left(\mathfrak{p}^{n}, F^{\prime} / K\right)\right\}, \quad F^{\prime}:=K\left(\mathfrak{f} \overline{\mathfrak{p}}^{\infty}\right) .
$$

注 2 这个定理是文献 [2] 中的定理 4.14 (限制特征的型满足 $0 \leqslant-j<k$ ) 结合推论 6.7 所得. 需 要注意的是 de Shalit 的结果中 $L$ - 函数的 Gamma 因子等于 $(2 \pi)^{-1}$, 那是由于他所用的 archimedean 周期等于 $(2 \pi)^{-1} \Omega_{\infty}$ (比较文献 [2] 中的定理 4.11 和 4.12).

注 3 在文献 $[3,4]$ 中我们定义和研究了一种本原的 $p$-adic $L$ - 函数, 从另外一个角度刻画这个解 析的对象, 并同样可以建立其与算术对象 Selmer 群之间的联系.

\section{Selmer 群和增大的 Selmer 群}

在本节我们假设 $F$ 是一个数域.

定义 对于 $\mathfrak{p}^{n}=(\alpha)$ 我们定义增大的 Selmer 群 $S_{\alpha}^{\prime}(E / F)$ 如下:

$$
S_{\alpha}^{\prime}(E / F)=\operatorname{ker}\left\{H^{1}(F, E[\alpha]) \rightarrow \prod_{v \nmid \mathfrak{p}} H^{1}\left(F_{v}, E\right)\right\} .
$$

引理 5 设 $\mathfrak{p}$ 是 $K$ 的一个素理想, $n(\mathfrak{p})$ 是最小的正整数使得 $1+\mathcal{O}_{\mathfrak{p}}^{n(\mathfrak{p})}$ torsion-free. 设 $n \geqslant n(\mathfrak{p})$, $E\left[\mathfrak{p}^{n}\right] \subset E(F)$ 并且 $\mathfrak{p}^{n}=\alpha \mathcal{O}$. 则我们有

$$
S_{\alpha}^{\prime}(E / F)=\operatorname{Hom}\left(\operatorname{Gal}(M / F), E\left[\mathfrak{p}^{n}\right]\right),
$$

这里 $M$ 是 $F$ 的在 $\mathfrak{p}$ 之外不分歧的极大交换 $p$ - 扩张.

证明 因为 $E\left[\mathfrak{p}^{n}\right] \subset E(F)$, 所以我们有上同调群的刻画:

$$
H^{1}\left(F, E\left[\mathfrak{p}^{n}\right]\right)=\operatorname{Hom}\left(G_{F}, E\left[\mathfrak{p}^{n}\right]\right), H^{1}\left(F_{v}, E\left[\mathfrak{p}^{n}\right]\right)=\operatorname{Hom}\left(G_{F_{v}}, E\left[\mathfrak{p}^{n}\right]\right),
$$

这里 $v$ 是不整除 $\mathfrak{p}$ 的素元. 所以 $E\left(F_{v}\right) / \alpha E\left(F_{v}\right)$ 包含在 $\operatorname{Hom}\left(G_{F_{v}} / I_{v}, E\left[\mathfrak{p}^{n}\right]\right)$ 中, 并且:

$$
\operatorname{Hom}\left(G_{F_{v}} / I_{v}, E\left[\mathfrak{p}^{n}\right]\right) \cong E\left[\mathfrak{p}^{n}\right] .
$$

结合 $E\left(F_{v}\right) / \alpha E\left(F_{v}\right) \cong E\left[\mathfrak{p}^{n}\right]$ 我们得到 $E\left(F_{v}\right) / \alpha E\left(F_{v}\right)$ 到 $H^{1}\left(F_{v}, E\left[\mathfrak{p}^{n}\right]\right)$ 的像恰好等于

$$
\operatorname{Hom}\left(G_{F_{v}} / I_{v}, E\left[\mathfrak{p}^{n}\right]\right),
$$

这样根据增大的 Selmer 群 $S_{\alpha}^{\prime}(E / F)$ 的定义就完成了引理的证明.

定理 6 令 $w_{H}$ 表示 $K$ 的类域 $H$ 中单位根的个数, $\mathcal{D}_{F / K}$ 表示 $F / K$ 的判别式. 设 $\mathfrak{p}$ 是 $K$ 的一 个素理想, 与 $w_{H} \mathcal{D}_{F / K}$ 互素并且满足 $\mathfrak{p}^{n}=\alpha \mathcal{O}$. 令 $F_{n}=F\left(E\left[\mathfrak{p}^{n}\right]\right)$. 则

$$
S_{\alpha}^{\prime}(E / F)=\operatorname{Hom}\left(\operatorname{Gal}\left(M_{n} / F_{n}\right), E\left[\mathfrak{p}^{n}\right]\right)^{\operatorname{Gal}\left(F_{n} / F\right)},
$$


这里 $M_{n}$ 是 $F_{n}$ 的在 $\mathfrak{p}$ 之外不分歧的极大交换 $p$ - 扩张.

证明 令 $G=\operatorname{Gal}\left(F_{n} / F\right)$, 由限制映射我们得到如下同构:

$$
H^{1}\left(F, E\left[\mathfrak{p}^{n}\right]\right) \cong H^{1}\left(F_{n}, E\left[\mathfrak{p}^{n}\right]\right)^{G} .
$$

显然在这个限制映射下 $S_{\alpha}^{\prime}(E / F)$ 的像包含在 $S_{\alpha}^{\prime}\left(E / F_{n}\right)$ 中. 相反地, 由于限制映射

$$
H^{1}\left(F_{v}, E\right) \rightarrow H^{1}\left(F_{v}\left[E\left[\mathfrak{p}^{n}\right]\right], E\right)
$$

对于每一个不整除 $\mathfrak{p}$ 的素元 $v$ 都是单射, 所以 $H^{1}\left(F, E\left[\mathfrak{p}^{n}\right]\right)$ 中那些通过限制映射落在 $S_{\alpha}^{\prime}\left(E / F_{n}\right)$ 中的 类同样也落在 $S_{\alpha}^{\prime}(E / F)$ 中, 从而得到

$$
S_{\alpha}^{\prime}(E / F)=S_{\alpha}^{\prime}\left(E / F_{n}\right)^{G},
$$

最后通过上述引理 5 完成证明.

定理 7 设 $\mathfrak{P}$ 是 $F$ 的在 $\mathfrak{p}$ 上的一个素元, $\xi=\psi(\mathfrak{P}), \hat{E}$ 表示椭圆曲线 $E$ 对应的形式群, $\lambda$ 是对 数映射

若 $\mathfrak{p}$ 与 $\mathcal{D}_{F / K}$ 互素并且 $E$ 在 $\mathfrak{p}$ 处有好的约化, 那么 $\hat{E}$ 是一个关于不分歧扩张 $F_{\mathfrak{P}} / K_{\mathfrak{p}}$ 和 $\xi$ 的相 对 Lubin-Tate 形式群. 当 $\mathfrak{p}$ 分裂的时候高度为 1 , 否则高度为 2 .

若 $E$ 在 $\mathfrak{p}$ 处有好的约化, 则 $E\left(F_{\mathfrak{P}}\right) / E_{1}\left(F_{\mathfrak{P}}\right)$ 没有 $\xi$-torsion 元. 若 $E$ 在 $\mathfrak{p}$ 处是坏的约化则 $E_{0}\left(F_{\mathfrak{P}}\right) / E_{1}\left(F_{\mathfrak{P}}\right)$ 同构于 $\mathcal{O}_{\mathfrak{P}} / \mathfrak{P} \mathcal{O}_{\mathfrak{P}}$ 并且 $E\left(F_{\mathfrak{P}}\right) / E_{0}\left(F_{\mathfrak{P}}\right)$ 是一个被 $w_{K}$ 零化的有限群.

映射 $\lambda$ 诱导了从 $E\left(F_{\mathfrak{P}}\right)$ 到 $F_{\mathfrak{P}}$ 的一个同态, 核为 $E\left(F_{\mathfrak{P}}\right)_{\text {torsion }}$, 同态像则如下描述:

$\mathfrak{P}^{j+1} \mathcal{O}_{\mathfrak{P}}$, 如果 $E$ 在 $\mathfrak{p}$ 处有好的约化, 并且 $\#\left(E\left(F_{\mathfrak{P}}\right)_{\mathfrak{p} \infty}\right)=\mathrm{NP}^{j}$.

$\mathfrak{P}^{j} \mathcal{O}_{\mathfrak{P}}$, 如果 $E$ 在 $\mathfrak{p}$ 处有坏的约化, $\mathfrak{p} \nmid w_{K}$, 并且 $\# E\left(F_{\mathfrak{P}}\right)_{\mathfrak{p} \infty}=\mathrm{NP}^{j}$.

$\mathfrak{P}^{j} \mathcal{O}_{\mathfrak{P}}$, 如果 $E$ 在 $\mathfrak{p}$ 处有坏的约化, $\mathfrak{p} \mid w_{K}$, 并且 $\#\left(E\left(F_{\mathfrak{P}}\right)_{\mathfrak{p} \infty}\right) / \#\left(\left(E\left(F_{\mathfrak{P}}\right) / E_{0}\left(F_{\mathfrak{P}}\right)\right)_{\mathfrak{p} \infty}\right)=\mathrm{N}^{j}$.

证明 证明类似于文献 [5], 关于 $\hat{E}$ 的性质可以参见文献 [2, 第 2 章第 1 节]. 对数映射 $\lambda$ 将 $\hat{E}\left(\mathfrak{P}^{n} \mathcal{O}_{\mathfrak{P}}\right)$ 同构地映成了 $\mathfrak{P}^{n} \mathcal{O}_{\mathfrak{P}}$. 因为 $E\left(F_{\mathfrak{P}}\right) / \hat{E}\left(\mathfrak{P}^{n} \mathcal{O}_{\mathfrak{P}}\right)$ 是一个 torsion 群, 对数映射唯一的拓展成为 从 $E\left(F_{\mathfrak{P}}\right)$ 到 $F_{\mathfrak{P}}$ 的映射, 并且核为 $E\left(F_{\mathfrak{P}}\right)_{\text {torsion }}$, 同态像为 $\mathfrak{P}^{s} \mathcal{O}_{\mathfrak{P}}$, 这里我们有

$$
E\left(F_{\mathfrak{P}}\right) /\left(E\left(F_{\mathfrak{P}}\right)_{\text {torsion }}+\hat{E}\left(\mathfrak{P}^{n} \mathcal{O}_{\mathfrak{P}}\right)\right) \cong \mathfrak{P}^{s} \mathcal{O}_{\mathfrak{P}} / \mathfrak{P}^{n} \mathcal{O}_{\mathfrak{P}} .
$$

从而得到

$$
E\left(F_{\mathfrak{P}}\right):\left(E\left(F_{\mathfrak{P}}\right)_{\text {torsion }}+\hat{E}\left(\mathfrak{P}^{n} \mathcal{O}_{\mathfrak{P}}\right)\right)=\frac{\left[E\left(F_{\mathfrak{P}}\right): E_{0}\left(F_{\mathfrak{P}}\right)\right]\left[E_{0}\left(F_{\mathfrak{P}}\right): E_{1}\left(F_{\mathfrak{P}}\right)\right]\left[\hat{E}\left(\mathfrak{P} \mathcal{O}_{\mathfrak{P}}\right): \hat{E}\left(\mathfrak{P}^{n} \mathcal{O}_{\mathfrak{P}}\right)\right]}{\# E\left(F_{\mathfrak{P}}\right)_{\text {torsion }}} .
$$

再利用如下事实: $\hat{E}\left(\mathfrak{P}^{k} \mathcal{O}_{\mathfrak{P}}\right) / \hat{E}\left(\mathfrak{P}^{k+1} \mathcal{O}_{\mathfrak{P}}\right) \cong \mathcal{O}_{\mathfrak{P}} / \mathfrak{P} \mathcal{O}_{\mathfrak{P}}$, 结合我们关于 $j$ 的定义, 从而得到上述等式的 右边是 $\#\left(E\left(F_{\mathfrak{P}}\right) / E_{0}\left(F_{\mathfrak{P}}\right)\right)_{\mathfrak{P}_{\infty}} \#\left(E_{0}\left(F_{\mathfrak{P}}\right) / E_{1}\left(F_{\mathfrak{P}}\right)\right)_{\mathfrak{P}_{\infty}} \mathrm{NP}^{n-1-j}$. 证毕.

\section{参考文献}

1 Rubin K. On the main conjecture of Iwasawa theory for imaginary quadratic fields. Invent Math, 1988, 93: 25-68

2 de Shalit E. Iwasawa Theory of Elliptic Curves with Complex Multiplication. Perspectives in Mathematics, vol. 3. London: Acdemic Press INC, 1987

3 Kang Y. On two-variable primitive $p$-adic L-functions. Asian J Math, 2012, 16: 171-188 
4 Kang Y L, Qin H L. On the $\mu$-invariant of two-variable primitive $p$-adic $L$-functions. Sci China Math, 2014, 57: $1149-1154$

5 Rubin K. Tate-Shafarevich groups and $L$-functions of elliptic curves with complex multiplication. Invent Math, 1987, 89: $527-560$

\section{Some arithmetic properties of Gross curves}

\section{KANG YunLing}

Abstract We study the arithmetic properties of Gross curves, and establish the connection between enlarged Selmer groups and Galois groups of certain unramified field extensions. We also study the structure of the rational points of the elliptic curves over local fields.

Keywords elliptic curves, complex multiplication, Iwasawa theory

MSC(2010) 11R23, 11G40

doi: 10.1360/N012014-00244 